\title{
Modified BLUE Protocol Ultrasonography can diagnose thrombotic manifestations of COVID-19 with Normal lung Ultrasound
}

\author{
Tamer Zaalouk ${ }^{1}$, zouheir Bitar ${ }^{1}$, Osama Maadarani ${ }^{1}$, and Ragab Elshabasy ${ }^{2}$ \\ ${ }^{1}$ Ahmadi Hospital \\ ${ }^{2}$ Kuwait Oil Company
}

September 11, 2020

\begin{abstract}
COVID-19 infection can present with thrombotic manifestations like PE with no ultrasonographic evidence of lung parenchymal affection. BLUE protocol provides excellent step by step approach for diagnosis of acute dyspnoea. Adding FECHO to BLUE protocol complete the picture and help making solid diagnosis especially in sub-massive and massive $\mathrm{PE}$
\end{abstract}

Modified BLUE Protocol Ultrasonography can diagnose thrombotic manifestations of COVID19 with Normal lung Ultrasound

Key Clinical message BLUE protocol provides excellent step by step approach for diagnosis of acute dyspnea. Adding F

\section{Case report}

71-year-old-woman with past history of Chronic kidney disease, presented to ER with acute dyspnea for 3 hours duration. Patient had history of low-grade fever 3 days before presentation, no cough, no expectoration and no Hemoptysis. Patient was fully conscious, but tachypneic (Respiratory rate $24 \mathrm{breath} / \mathrm{min}$ ), pulse oximetry showed $90 \%$ on room air. Blood pressure $90 / 50 \mathrm{mmHg}$ and heart rate $110 \mathrm{~b} / \mathrm{min}$. Chest Xray was normal, Electrocardiography (ECG) show sinus tachycardia with new right bundle branch block (figure 1).

Arterial blood gas analysis in ambient air confirmed type 1 respiratory failure ( $\mathrm{PaO} 29.4 \mathrm{kPa})$. As a case of acute dyspnea bed side BLUE ultrasound protocol applied where there was normal lung sliding with A-profile of lung Ultrasound (figure 2), no B-lines and no any sub-pleural consolidations.

According to BLUE protocol sequences we proceeded to assess lower limb veins and unfortunately there was bilateral femoral vein thrombosis. At this point the main differential diagnosis was pulmonary embolism (PE), We modified the BLUE protocol by adding Focused cardiac ultrasound (FECHO) assessment, that confirm the diagnosis of $\mathrm{PE}$ as it shows Right ventricular dilation, Positive McConnell's sign and pulmonary hypertension (Pulmonary artery systolic pressure equal $50 \mathrm{mmHg}$ ). Such LUS and FECHO findings during the current pandemic raised the suspicion of COVID-19 infection as a cause of the patient's clinical presentation.

Laboratory findings were significant for mild leucocytosis with lymphopenia, raised D-dimer, troponin I and pro-BNP, and evidence of acute kidney injury.

\section{Laboratory results}


WBCs (White blood count) $=15,000$

WBCs $($ White blood count $)=15,000 \mathrm{HB}($ Haemoglobin $)=13.4$ grams Platelets $=103,000$ Urea $=15$ milligram $/ \mathrm{dl}$ Creatin

\section{Discussion}

Acute dyspnoea could be a common symptom within the ED. the quality approach to dyspnoea often relies on radiologic and laboratory results, causing excessive delay before adequate therapy is started. Use of an integrated point-of-care ultrasonography (POCUS) approach can shorten the time needed to formulate a diagnosis, while maintaining a suitable safety profile (1).

Chest computerized tomography (CT) has significant limitations, like exposure to ionized radiation, limited application in certain patients, like pregnant women, the need of transferring a potentially unstable patient to the tomography unit and time consuming (2).

POCUS is employed by physicians at the bedside for rapid, focused and accurate evaluation to spot or rule out various pathologies. Several protocols for POCUS are currently available and employed in different clinical scenarios like undifferentiated dyspnoea, hypoxia or shock, and include the bedside lung ultrasound in emergency (BLUE), the rapid assessment of dyspnoea with ultrasound (RADIUS) and also the rapid ultrasound in shock (RUSH) protocols (3).

The bedside lung ultrasonography in emergency (BLUE) protocol is an algorithm developed by Lichtenstein as a systemic approach to the diagnosis of patients with dyspnoea in critical care units (ICUs) with $90.5 \%$ diagnostic accuracy. A (BLUE) protocol provides good step by step approach to diagnose acute dyspnoea (4).

The BLUE protocol started by checking Anterior lung sliding. Presence of sliding exclude pneumothorax. The B profile suggests pulmonary oedema. The A profile prompts an enquiry for thrombosis. The association of A profile with phlebothrombosis (venous scan) favours the diagnosis of pulmonary embolism with $81 \%$ sensitivity and $99 \%$ specificity (5).

At this point, adding a focus ECHO (FECHO) examination to BLUE protocol can confirm presence of embolism (PE) especially if it's massive or sub-massive PE. Ultrasound signs of right heart strain include bowing of the IVS into the LV, right ventricular dilatation and systolic dysfunction including McConnell's sign, possible tricuspid regurgitation, a dilated inferior vena cava and visual right heart thrombus (6).

Coronavirus disease 2019 (COVID-19) is caused by severe acute respiratory syndrome coronavirus 2 (SARSCoV-2), the World Health Organization (WHO) declared it an pandemic on 11 March 2020 (7).

The effect of SARS-CoV-2 on endothelial cells plays a vital role in vascular injury that contributes to pulmonary, cardiovascular and other manifestations. Endothelial dysfunction and endothelitis are considered the idea of thrombus formation and lead to COVID-19 associated thromboembolic insult of various organs and might partially explain the hypercoagulable state commonly related to patients infected with SARS-CoV-2 (7).

Thromboembolic manifestations of COVID-19 have been described in several reports. In the ED and critical care units, BLUE protocol plus FECHO in the appropriate clinical context is an effective tool to rapidly diagnose acute pulmonary embolism associated with right heart strain and possible thrombus in transit, and guide further treatment (8).

In our case the patient presented with acute dyspnoea with no clinical symptoms or signs that favour diagnosis of (COVID-19), using an Extended BLUE protocol by adding FECHO help in diagnosis acute massive PE with bilateral lower limb DVT. within the context of the present Pandemic with thrombosis everywhere, we diagnose this patient as (COVID-19) infection. Which was confirmed by positive nasopharyngeal swab for SARS-CoV-2 shortly. 
We stress on the importance of BLUE protocol for assessment of patient with acute dyspnoea and recommend to include FECHO examination for more accurate diagnosis.

\section{Conclusion}

COVID-19 infection can present with thrombotic manifestations like DVT and PE with no ultrasonographic evidence of lung parenchymal affection. BLUE protocol provides excellent step by step approach for diagnosis of acute dyspnea. Adding FECHO to BLUE protocol complete the picture and help making solid diagnosis especially in sub-massive and massive PE.

\section{Disclaimers}

The author(s) declared no potential conflicts of interest with respect to the research, authorship, and/or publication of this article.

\section{Funding}

The authors received no financial support for the research, authorship, and/or publication of this article. The research was performed as part of the employment of the authors in Kuwait oil Company

\section{Contribution}

Tamer Zaalouk wrote the article, Zouheir Ibrahim Bitar and Ossama S. Maadarani shared in the discussion and with ALAsmar Mohammed El-shably

in collecting the data and revision of the manuscript

Our working web site iswww.kockw.com Kuwait 0il company, Ahamdi hospital

\section{References}

1. Zaalouk, TM, Bitar, ZI, Maadarani, OS, El-shably, AM. White lung with milky effusion. Clin Case Rep . 2020; 00: 1- 4. https://doi.org/10.1002/ccr3.3207

2. Bitar et al. Ann. Intensive Care (2015) 5:56

3. Zanobetti M, Poggioni C, Pini R. Can chest ultrasonography replace standard chest radiography for evaluation of acute dyspnea in the ED? Chest 2011;139:1140-7.Macfarlane JR, Holman CW. Chylothorax. Am Rev Respir Dis 1972; 105:287.

4. Volpicelli G, Elbarbary M, Blaivas M, Lichtenstein DA, Mathis G, Kirkpatrick AW, et al. International evidence-based recommendations for point-of-care lung ultrasound. Intensive Care Med 2012;38 (4):577-591.

5. Lichtenstein DA, Mezière GA. Relevance of lung ultrasound in the diagnosis of acute respiratory failure: the BLUE protocol. Chest 2008;134:117-25.

6. Bryant AS, Minnich DJ, Wei B, Cerfolio RJ. The incidence and management of postoperative chylothorax after pulmonary resection and thoracic mediastinal lymph node dissection. Ann Thorac Surg 2014; 98:232.

7. Maadarani O, Bitar Z, Zaalouk T, Mohsen M Elshabasy R. Point-of-care ultrasound can suggest COVID-19. EJCRIM 2020;7 : doi:10.12890/2020_001915.

8. Kahl N, Gabriel C, Lahham S, Thompson M, Hoonpongsimanont W. Point-of-care ultrasound diagnosis of pulmonary embolism with thrombus in transit. Clin Pract Cases Emerg Med 2019;3 (1):11-12. Doerr CH, Allen MS, Nichols FC 3rd, Ryu JH. Etiology of chylothorax in 203 patients. Mayo Clin Proc 2005;80:867.

Figures 


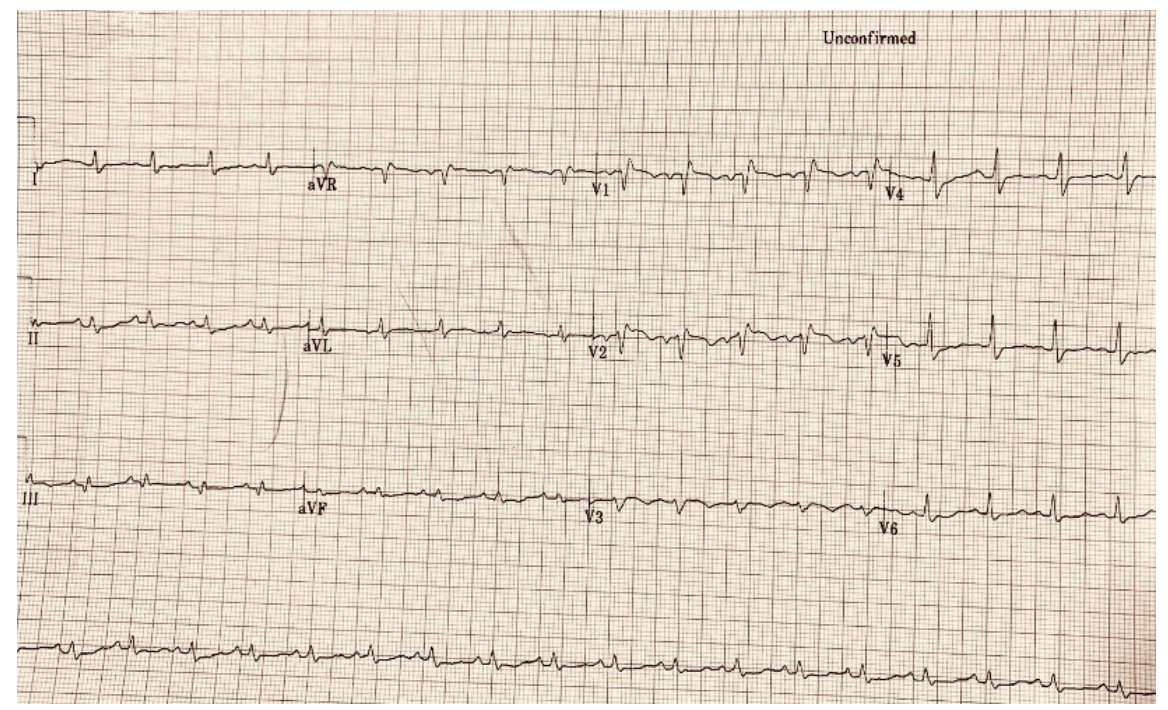

Figure (1): Electrocardiography (ECG) show sinus tachycardia and RBBB

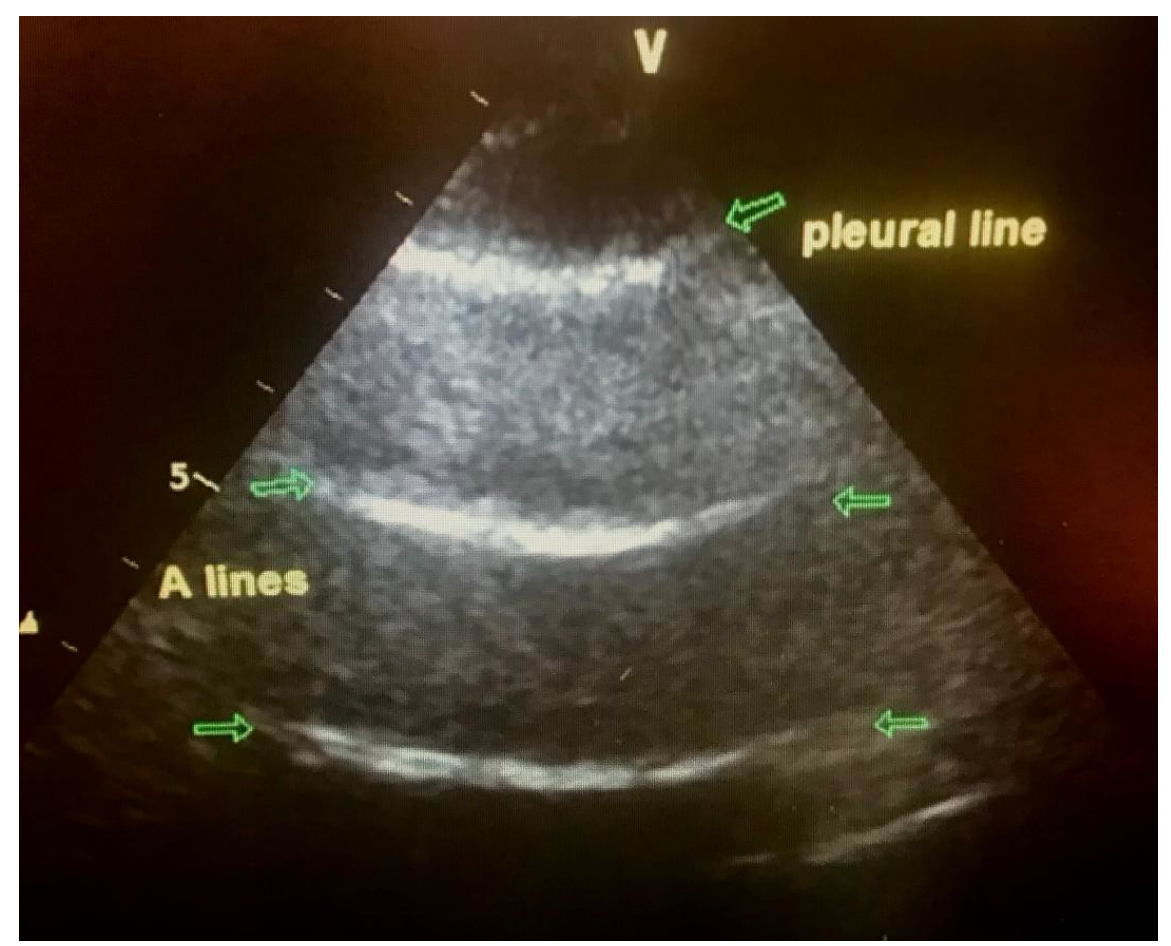

Figure (2): A-profile lung 


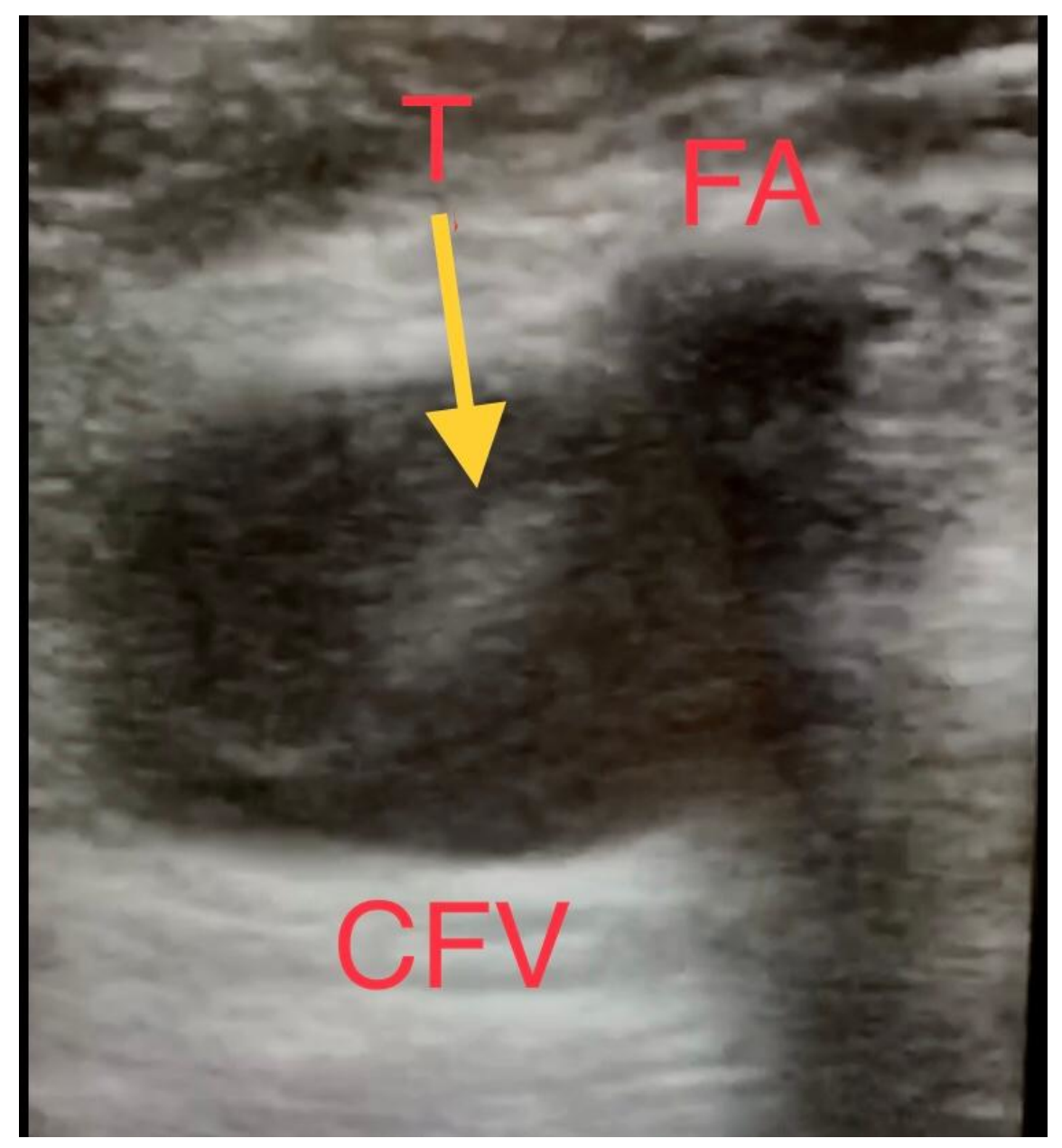

Figure (3): Doppler venous system of non-compressible right common femoral vein- partial thrombosis $(\mathrm{T})$

CFV- Common femoral vein, FA- Femoral artery, T- Thrombus 


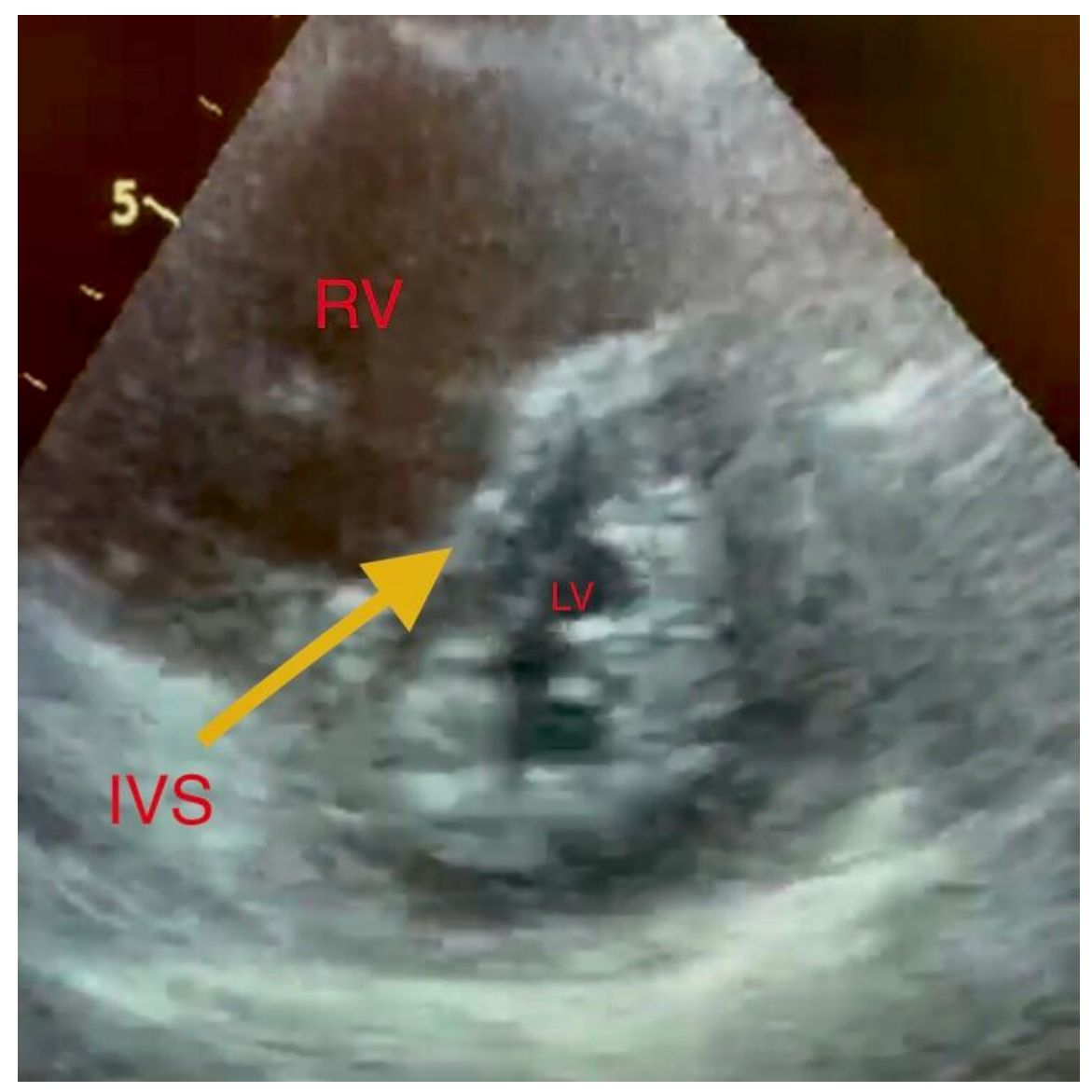

Figure (4): parasternal short axis view show dilated right ventricle (RV) with shifted interventricular septum (IVS) toward small left ventricle (LV) 


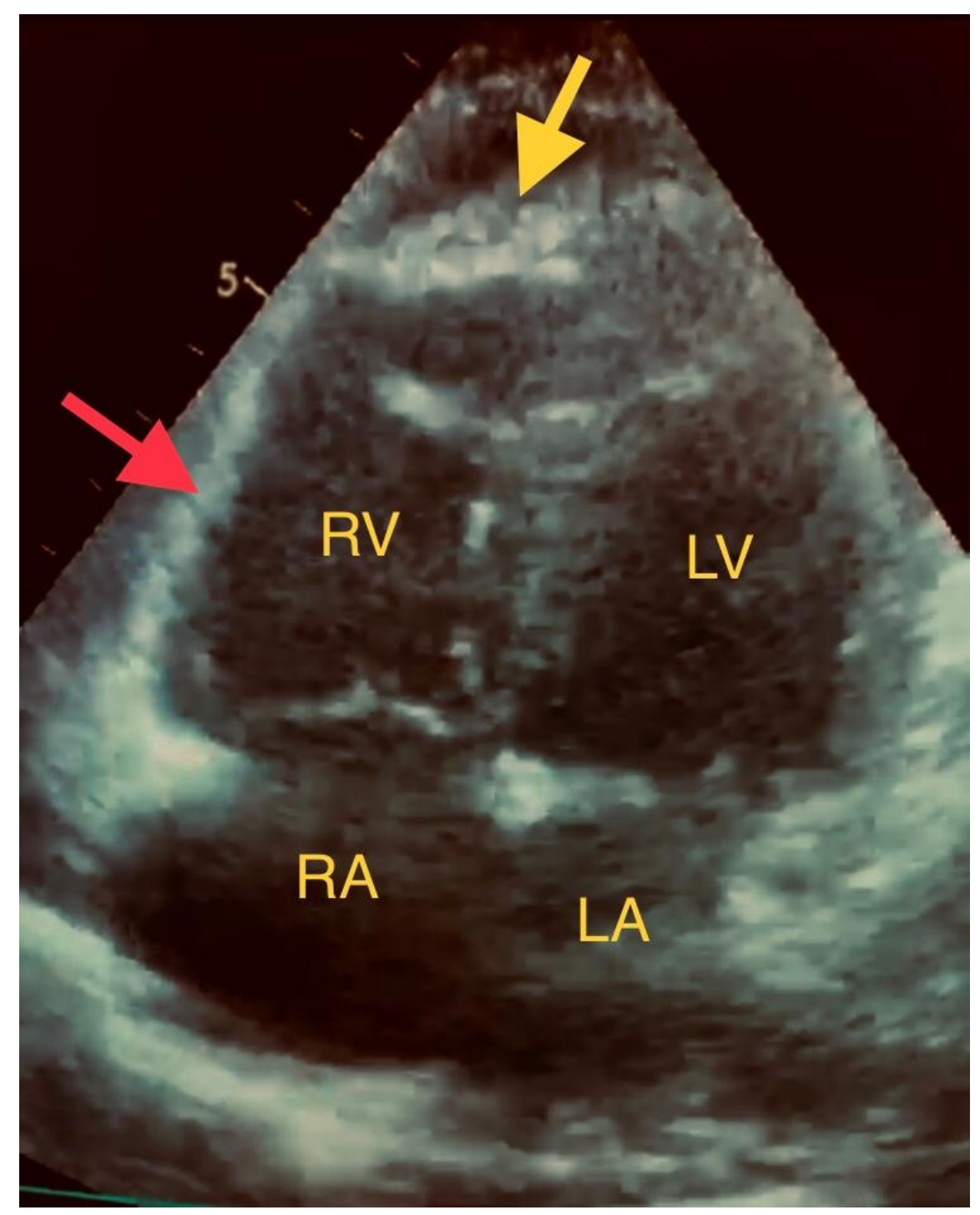

Figure (5): McConnell's sign : Akinesia of mid free wall of right ventricle (red arrow) with normal motion at the apex (yellow arrow). RV- Right ventricle, LV - Left ventricle, RA- Right atrium, LA-Left atrium

\section{Hosted file}

figures.docx available at https://authorea.com/users/349297/articles/479978-modified-blueprotocol-ultrasonography-can-diagnose-thrombotic-manifestations-of-covid-19-with-normallung-ultrasound 